\title{
Flow cytometric sorting coupled with exon capture sequencing identifies somatic mutations in archival lymphoma tissues
}

\author{
Nenggang Jiang ${ }^{1,2}$, Christopher Chen ${ }^{3}$, Qiang Gong ${ }^{2}$, Kristen Shields ${ }^{4}$, Yuping Li ${ }^{2}$, YuanYuan Chen², Joo Song ${ }^{2}$, \\ Timothy W McKeithan ${ }^{2}$ and Wing C Chan ${ }^{2}$
}

The enormous number of archived formalin-fixed paraffin-embedded (FFPE) tissues available are a valuable resource of material for research. However, the use of such tissues poses many challenges, among which is the difficulty of isolating different cell populations within the tissue. In this study, we used tissue from two types of non-Hodgkin lymphoma as a model to demonstrate a method we have established and optimized to separate FFPE samples into distinct tumor and nonmalignant populations. Using FFPE reactive tonsil sections, various approaches for antigen retrieval and labeling, and the effectiveness of flow cytometric sorting were tested. We found that, among the 11 cell surface or intracellular antigen markers investigated, CD3ع, CD79A, LAT, PD-1, and PAX5 could be successfully labeled after antigen retrieval in Tris-EDTA buffer ( $\mathrm{pH}$ 8.0) at $65^{\circ} \mathrm{C}$ for $60 \mathrm{~min}$, and 1.8-2.7 $\mu \mathrm{g}$ DNA per million cells could be extracted after sorting with DNA quality similar to that of tissue without staining or sorting. To test whether we could perform next-generation sequencing using a custom capture platform on sorted cells, we used three lymphoma cases with FFPE tissues which had been stored for 1 to 4 years. We demonstrated that the DNA from sorted cells was adequate for exon capture sequencing. By comparing the sequencing results between neoplastic and normal populations, somatic mutations could be clearly identified in the tumor population with variant frequencies as low as $11.7 \%$. The corresponding normal fraction clearly helps in the analysis of somatic mutations and the exclusion of artifacts. This study provides an approach using flow cytometric sorting to separate different cellular populations in paraffin-embedded tissues and to unambiguously distinguish somatic mutations from germline variants or artifacts. This approach is also useful in enriching the tumor component in samples with heterogeneous components and low tumor content.

Laboratory Investigation (2017) 97, 1364-1374; doi:10.1038/labinvest.2017.73; published online 7 August 2017

Formalin-fixed paraffin-embedded (FFPE) tissues are routinely utilized in lymphoma research, ${ }^{1}$ as this preservation process is both ubiquitous and robust. However, the use of FFPE tissues for antigen-antibody labeling and nextgeneration sequencing (NGS) has consistently posed logistical challenges. First, the preservation process induces crosslinks that mask epitopes for antibodies. Heating FFPE samples to temperatures near $100^{\circ} \mathrm{C}$ is often recommended for crosslink reversal and antigen retrieval. However, using such high temperatures for antigen retrieval may damage DNA and reduce its quality to the extent that it may no longer be suitable for downstream applications such as exon capture sequencing.
An additional difficulty of conducting sequencing analysis on lymphoma samples is the frequent absence of the corresponding normal samples and the heterogeneity of cells contained within the biopsied tissue. ${ }^{2}$ Although many germline variants that match known variants present in the general population can be eliminated by comparison to databases such as the single nucleotide polymorphism database (dbSNP), rare germline variants may be missed in the absence of a corresponding normal sample. In order to have confidence of the somatic origin of the detected single nucleotide variants (SNVs) or other apparent mutations, NGS requires both the neoplastic cells and a corresponding sample of normal, non-neoplastic cells with which to compare. ${ }^{3}$

\footnotetext{
${ }^{1}$ Department of Laboratory Medicine, West China Hospital, Sichuan University, Chengdu, China; ${ }^{2}$ Department of Pathology, City of Hope National Medical Center, Duarte, CA, USA; ${ }^{3}$ College of Chemistry, UC Berkeley, Berkeley, CA, USA and ${ }^{4}$ College of Osteopathic Medicine of the Pacific, Western University of Health Sciences, Pomona, CA, USA

Correspondence: Dr WC Chan, MD, Department of Pathology, City of Hope National Medical Center, Room 1215, 1500 East Duarte Road, Duarte, CA 91010, USA. E-mail: jochan@coh.org
} 
A further problem is that the fraction of neoplastic cells is low in some non-Hodgkin lymphoma samples, resulting in a low variant allele frequency (VAF). In such cases the mutations may be entirely missed or present at such low frequencies as to be difficult to distinguish from background sequencing errors. ${ }^{4}$

Purification of tumor lymphocytes by flow sorting or magnetic separation can help to resolve these problems, but these procedures are usually done on freshly isolated cells. ${ }^{5-7}$ For FFPE tissue, laser microdissection can be utilized, ${ }^{8-12}$ but the technique is labor-intensive, and it is difficult to obtain a sufficient number of cells. Furthermore, prior labeling of the tissue sections may be necessary to facilitate the recognition of the desired cell populations to isolate, and routine antigen retrieval procedures may further degrade the DNA.

It is clearly of value to optimize a protocol for flow sorting and mutational analysis that separates samples into neoplastic and corresponding normal cells after antigen retrieval and immunostaining without compromising the integrity or DNA quality of the single cells. A few previous research reports have mentioned the value of isolating tumor cells for cancer studies on FFPE tissue through DNA ploidy and immunophenotyping analysis. ${ }^{13,14}$ However, these studies did not provide a robust protocol for ensuring effective antibody labeling and the isolation of DNA of sufficient quality for exon capture sequencing. In this study, we report a method for separating FFPE samples into distinct groups of neoplastic and nonneoplastic cells by gentle antigen retrieval, antigen labeling, and flow cytometric sorting. We confirmed the validity of our method by extracting DNA from the sorted FFPE cells and assessing it for quality. In addition, we performed exon capture sequencing on our enriched cell populations to determine whether our approach will help to identify tumorassociated mutations, especially those occurring in lymphomas with low tumor content.

\section{MATERIALS AND METHODS Materials}

Four de-identified FFPE tissue blocks from the archives of the Department of Pathology at City of Hope Medical Center were utilized in this research, including one tonsil tissue stored for two years with reactive hyperplasia that was used for evaluating the conditions for cell isolation and antigen retrieval and labeling, one lymph node sample stored for one and a half years from a patient with follicular lymphoma (FL), and two lymph node samples from patients with angioimmunoblastic T-cell lymphoma (AITL; named as case 1 and case 2, stored for one year and four years, respectively). These lymphoid tissues were fixed for $4 \mathrm{~h}$ to overnight in $10 \%$ buffered formalin before further processing to prepare FFPE blocks. The diagnoses were confirmed by hematopathologists in the department. This study was approved by the Institutional Review Board of the City of Hope Medical Center. Thick sections of 50 micrometers were prepared.

\section{Single Cell Isolation from FFPE Tissue Sections}

For estimating cell recovery, FFPE tissue sections, after removing the surrounding paraffin, were weighed with GeneMate GM Research Balance (B-1850-60). Then the tissue sections were deparaffinized three times with xylene in a $1.5 \mathrm{ml}$ Eppendorf tube, each with $10 \mathrm{~min}$ incubation and rotation at room temperature. This was followed by sequential $5 \mathrm{~min}$ rehydration in ethanol at decreasing concentrations, from $100 \%$ (twice) to $90,70,50$, and $20 \%$. Ethanol was washed out with water during the last step. Each time the tube was centrifuged at $10000 \mathrm{~g}$ for $3 \mathrm{~min}$ and the supernatant was discarded carefully.

The tissue was added to $150 \mu \mathrm{l}$ PBS in a $1.5 \mathrm{ml}$ Eppendorf tube and ground gently with a Fisherbrand ${ }^{\mathrm{TM}}$ Pellet Pestle (Fisher Scientific, catalog No. 12-141-364) into a crude cell suspension, which was then digested in $300 \mu \mathrm{l}$ PBS with a final concentration of $0.3 \%$ collagenase/dispase (Sigma-Aldrich, catalog No. 11097113001 ) at $37^{\circ} \mathrm{C}$ for $10 \mathrm{~min}$. Digestion was stopped immediately by adding EDTA to a final concentration of $5 \mathrm{mM}$, and the cell suspension was placed on ice. After that, the cell suspension was filtered using a $70 \mu \mathrm{m}$ nylon mesh (Fisher Scientific, catalog No. 22363548), followed by washing the filter with $10 \mathrm{ml}$ cold PBA (1\% BSA in PBS), and all filtrates were combined in a $50 \mathrm{ml}$ centrifuge tube (BioPioneer, catalog No. CNT-50). Then the cell suspension was centrifuged at $500 \mathrm{~g}$ for $5 \mathrm{~min}$, and the cell pellet was resuspended in $10 \mathrm{ml} \mathrm{PBA}$ and centrifuged again. After discarding the supernatant and resuspending the cell pellet in $1 \mathrm{ml} \mathrm{PBA}$ in an Eppendorf tube, cell concentration was estimated by Countess automated cell counter (Invitrogen). Single-cell recovery per milligram tissue was then calculated.

\section{Antigen Retrieval}

In order to ensure an adequate number of cells for sorting, we used $1 \times 10^{6}$ cells per tube. The cells were pelleted by centrifugation in an Eppendorf tube at $500 \mathrm{~g}$ for $5 \mathrm{~min}$, and the supernatant was discarded. Tris-EDTA buffer (Tris $10 \mathrm{mM}$, EDTA $1 \mathrm{mM}, \mathrm{pH} \mathrm{8.0)}$ was added, and the cells were treated with various incubation times and temperatures: $85^{\circ} \mathrm{C} / 25 \mathrm{~min}, \quad 85^{\circ} \mathrm{C} / 15 \mathrm{~min}, \quad 70^{\circ} \mathrm{C} / 80 \mathrm{~min}, \quad 70^{\circ} \mathrm{C} / 60 \mathrm{~min}$, $65^{\circ} \mathrm{C} / 60 \mathrm{~min}, \quad 55^{\circ} \mathrm{C} / 60 \mathrm{~min}, \quad 45^{\circ} \mathrm{C} / 60 \mathrm{~min}$, and $37^{\circ} \mathrm{C} / 60$ min. After centrifugation at $500 \mathrm{~g}$ for $5 \mathrm{~min}$ and removal of the supernatant, the cell pellet was resuspended in $400 \mu \mathrm{l}$ PBA and transferred to a $5 \mathrm{ml}$ Falcon tube (Life Sciences, catalog No. 352054).

\section{Immunostaining}

Before incubation with antibodies, Triton X-100 was added to a final concentration of $0.1 \%$. The cell suspension was mixed well and incubated at room temperature for $10 \mathrm{~min}$. The cells were then added to $4 \mathrm{ml} \mathrm{PBA}$ and resuspended in $400 \mu \mathrm{l}$ PBA after centrifugation at $500 \mathrm{~g}$ for $5 \mathrm{~min}$ and discarding the supernatant. Fluorescent-labeled or unlabeled primary antibodies were added to the cell suspension and incubated for 2 to $4 \mathrm{~h}$ in a dark box at room temperature. Following 
incubation, $4 \mathrm{ml}$ PBA was added into the Falcon tube, and the supernatant was discarded carefully after centrifugation at $500 \mathrm{~g}$ for $5 \mathrm{~min}$. The washing step was repeated twice in order to completely remove the unbound antibodies. Next, the cell pellet was resuspended in $400 \mu \mathrm{lBA}$, and fluorescent-labeled secondary antibody was added in some cases with incubation for 30 to $60 \mathrm{~min}$, followed by washing once with $4 \mathrm{ml}$ PBA and resuspension in $600 \mu \mathrm{l}$ PBA. Multiparameter flow cytometric analysis and cell sorting were performed using the BD Aria III cytometer with Diva software (BD Biosciences). The performance of the flow cytometer was monitored daily using CS\&T research beads (BD Biosciences, catalog No. 650621), and the drop delay was set by Accudrop Beads (BD Biosciences, catalog No. 345249).

We tested antibodies to $\mathrm{T}$ - and B-cell surface or intracellular molecules, including CD3, LAT (linker for activation of T-cells), BCL11B, CD4, CD8, PD1 (programmed death-1), CD20, CD79A, PAX5 (paired box 5), and CXCR5 for cell labeling and flow cytometric sorting; for CD3 labeling, both a monoclonal antibody (UCTH1) binding the ectodomain of CD3e and a polyclonal antibody binding its cytoplasmic domain were evaluated. Information regarding the antibody reagents used is listed in Table 1 .

Stained cells were gated into distinct populations, and 50000 to 200000 cells were sorted. CD79A or PAX5 positive and CD3 or LAT positive populations were defined as B-cells and T-cells, respectively. To confirm that these sorted populations did, in fact, represent B- or T-cells, the sorted cells were stained with other T-cell and B-cell specific markers, and the sample was then immunophenotyped to assess for purity.

\section{Genomic DNA Extraction and Sequencing}

The sorted cells were incubated with $180 \mu$ l tissue lysis buffer and $20 \mu \mathrm{l}$ proteinase $\mathrm{K}$ (QIAamp DNA FFPE Tissue Kit, catalog No. 56404) at $65^{\circ} \mathrm{C}$ for $16 \mathrm{~h}$ and then incubated for $4 \mathrm{~h}$ at $56^{\circ} \mathrm{C}$ following the addition of another $20 \mu \mathrm{l}$ proteinase K. DNA was then extracted following the remaining wash and elution steps of the kit. DNA concentration was estimated by NanoDrop through measuring the absorbance at $260 \mathrm{~nm}$.

The quality of the extracted DNA was assessed by $1 \%$ agarose gel electrophoresis and quantitative PCR (qPCR) assays with two primer sets, QD196 and QD295 (Table 2), that amplify products of 196 and $295 \mathrm{bp}$, respectively. Equal quantities of template for $\mathrm{qPCR}$ were added, and the relative quantities of amplified products were compared. As a control, DNA extracted from fresh cells was assayed simultaneously.

\section{Target Sequencing and Sequence Alignment}

NGS was performed on DNA determined to be of sufficient quality. The sequencing libraries were constructed according to the manufacturer's instructions. All coding exons of a panel of 334 genes (Supplementary Table 1) were captured using the Agilent SureSelect platform. Paired-end $(2 \times 100 \mathrm{bp})$ multiplex sequencing of the captured fragments was performed on the Illumina HiSeq2500 instrument.

Paired-end reads in FASTQ format were aligned to the human reference genome (build hg19) using the BurrowsWheeler Aligner $(B W A \text {; v0.7.5a })^{15}$ with default parameters.

\section{Table 2 Primers used in DNA quality assessment}

\begin{tabular}{ll}
\hline Name & Sequence \\
\hline QD196-forward & 5'-CATGGCTCACTGGCTTACAA-3' \\
QD196-reverse & 5'-TTGCCTCTAACAGAGGAGCAG-3' \\
QD295-forward & 5'-GTCAGAAGACTGAAAACGAAGCC-3' \\
QD295-reverse & 5'-GCTTGCCACACTCTTCTTCAAGT-3' \\
\hline
\end{tabular}

Table 1 Lymphocyte related antibody reagents utilized in study

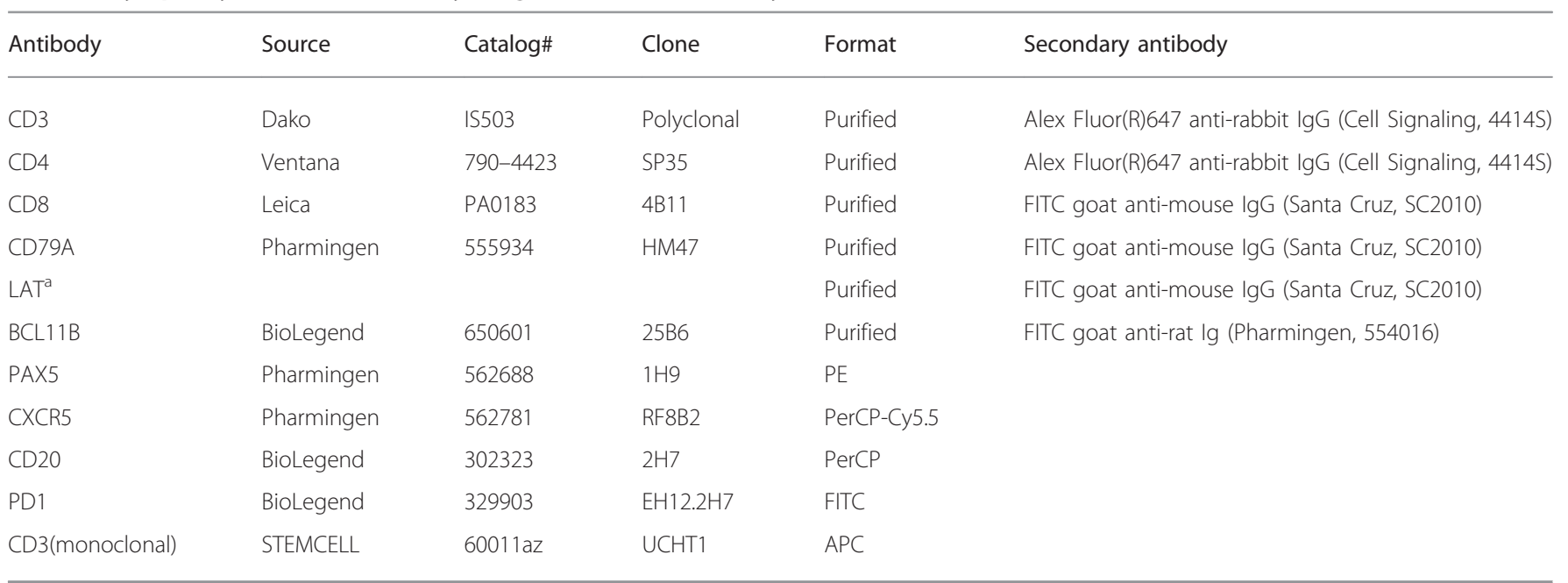

${ }^{a}$ Kind gift from Dr Pierre Broussett, Toulouse, France. 
Genome Analysis Toolkit (v3.1) ${ }^{16}$ was used to re-align the reads around known indels, and Picard (v1.115, Broad Institute, Cambridge, MA, USA) was used to mark the PCR duplicates.

\section{Variant Calling and Annotation}

Genomic variants were called using VarScan (v2.3.6). ${ }^{17}$ The following filtering criteria were applied to remove artifacts: (1) Only reads with mapping quality assigned by $B W A$ larger than 36 were used; (2) variant positions had to be covered by at least 10 reads, with the variant allele being supported by at least 4 reads; (3) the VAF had to be at least 5\% in at least one sample; and (4) variants with more than 90\% supporting reads from one strand were removed.

The variants were then annotated using ANNOVAR (version: 2015-12-14). ${ }^{18}$ Variants that were recorded in dbSNP database (build: 138) and had a non-reference population allele frequency larger than $1 \%$ were considered to be germline mutations and removed.

\section{RESULTS}

\section{Single Cell Preparation}

After disaggregating the FFPE tissues into single cell preparations, 1.5, 1.9, and 2.4 million cells were obtained per mg of FFPE lymphoid tissue with reactive hyperplasia in three experiments, and microscopic examination showed many single cells. The nuclei of all cells were stained by trypan blue due to disruption of the plasma membranes, but the cytoplasm of many cells was still visible (Figure 1).

\section{Cell Labeling and Flow Cytometric Sorting}

We assessed the labeling of different antigens for flow sorting after antigen retrieval with various temperature-time combinations. For identifying T-cells and B-cells, the antigens

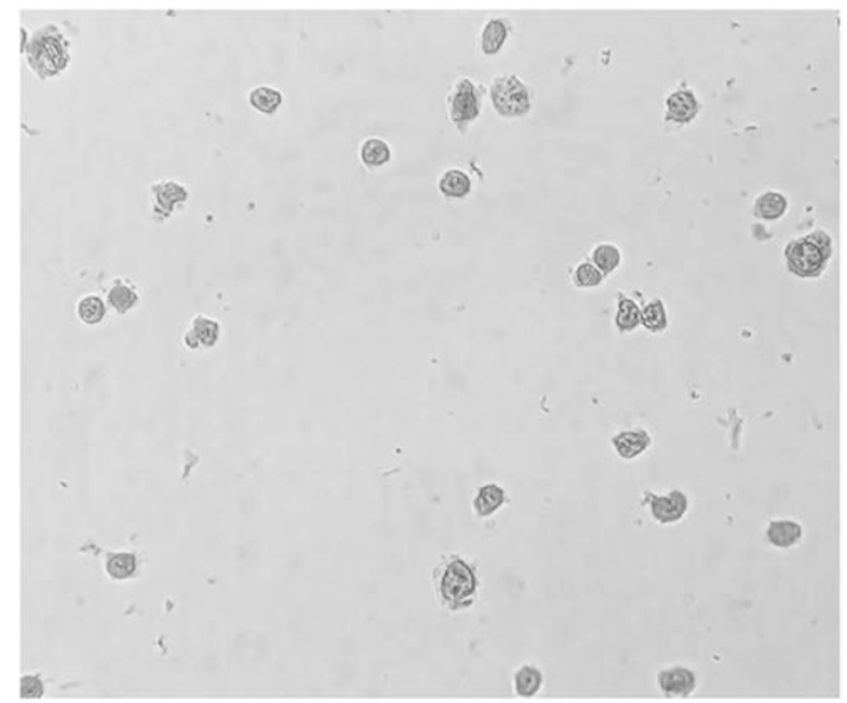

Figure 1 Cellular morphology in suspension after cell isolation from FFPE tissue ( $\times 40$, trypan blue stain, reactive hyperplasia tissue sample). targeted initially included two B-cell lineage-specific intracellular molecules (PAX5 and CD79A) and three T-cell lineagespecific intracellular molecules (CD3, BCL11B, and LAT). For $\mathrm{CD} 3$, two different antibodies were evaluated. Temperaturetime combinations ranged from $85^{\circ} \mathrm{C} / 25 \mathrm{~min}$ to $37^{\circ} \mathrm{C} /$ $60 \mathrm{~min}$.

Fluorescence was observed for four of the intracellular molecules of interest (CD3 (with polyclonal antibody), CD79A, LAT, and PAX5), showing that they were successfully labeled by their corresponding antibodies (Figure 2). However, the intensity of fluorescence varied depending on the antigen retrieval conditions used. A gradual reduction of fluorescence intensity was observed with decreasing time or temperature for antigen retrieval (Figure $2 \mathrm{a}-\mathrm{h}$ ).

Two of the antibodies to intracellular molecules specific to the T-cell lineage-antibody to BCL11B, a nuclear marker, and the monoclonal antibody (UCHT1) to CD3, an antigen expressed both on the plasma membrane and intracellularly -were not suitable for the separation of T-cells from B-cells, because BCL11B was found to be only partially positive in T-cells, while CD3 (UCHT1) was unable to label T-cells sufficiently from the FFPE tissues (Figure 3).

We also assessed the utility of membrane markers CD4, CD8, PD1, CXCR5, and CD20 (Figure 3). CD4 was found to be partially positive with low fluorescence, but there were not distinctly defined CD4-positive and -negative populations, limiting its utility in isolating T-cells. PD1 was found to be positive on a small population of cells in the case of reactive hyperplasia, but positive in a large subpopulation of cells in AITL cases (see below). The other antigens could not be labeled regardless of the conditions used, and these antigen labels were not pursued in subsequent experiments.

We compared the antigen retrieval results using Tris-EDTA and citrate buffer. There was no significant difference between the antigen retrieval effects of these two buffers using similar incubation conditions (Supplementary Figure 1). In addition, we found that using $0.3 \%$ collagenase/dispase digestion for $10 \mathrm{~min}$ when preparing single cell suspension did not result in a detectable decrease in the intensity of the cytoplasmic antigen markers we examined, but extending the incubation time to $30 \mathrm{~min}$ resulted in significantly lower CD79A staining (Supplementary Figure 2).

\section{DNA Quality Assessment after Sorting}

DNA was extracted from three types of cell samples: separated cells from FFPE reactive lymphoid tissue after antigen retrieval and sorting (based on our protocol described above), FFPE reactive lymphoid tissue without antigen retrieval, and cells from the Jurkat cell line (Clone E6-1, ATCC TIB-152) as control. The quality of extracted DNA was assessed and compared. Gel electrophoresis indicated that DNA derived from FFPE samples was generally of poor quality, with smears in the lanes, while high-molecular-weight DNA could be seen only on the lane with DNA from fresh cells (Figure 4). 
For qPCR detection, equal quantities $(5 \mathrm{ng})$ of all DNA samples were used as templates. The quantities of amplified products from the FFPE samples were compared to that from the fresh Jurkat cell line. We found that the amplified $196 \mathrm{bp}$ products from FFPE tissue were around $12 \%$ of those from a fresh culture of the Jurkat cells (Figure 4), presumably due to a

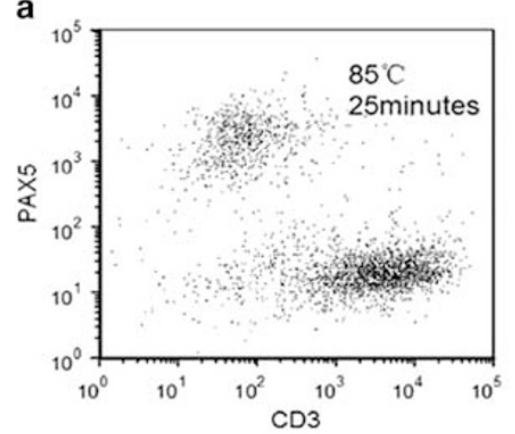

d

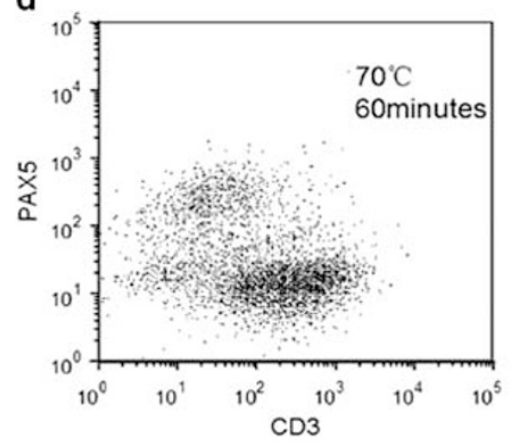

g

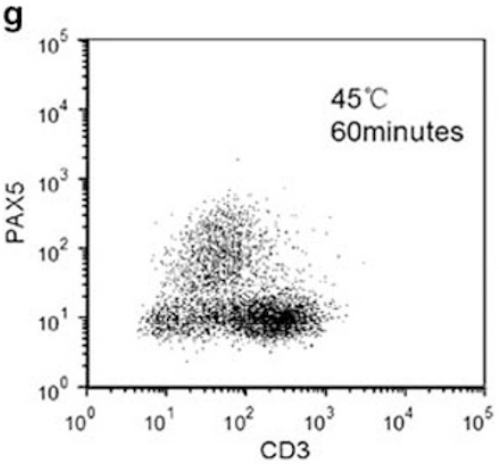

j

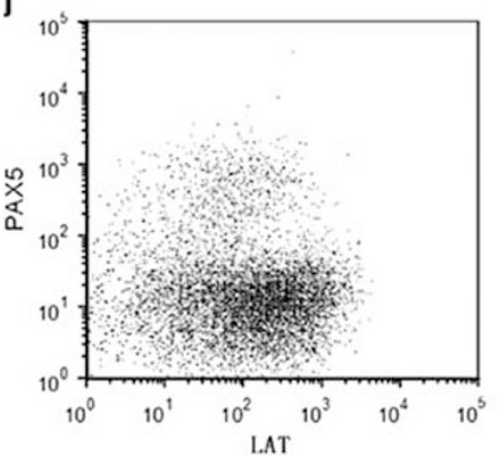

b

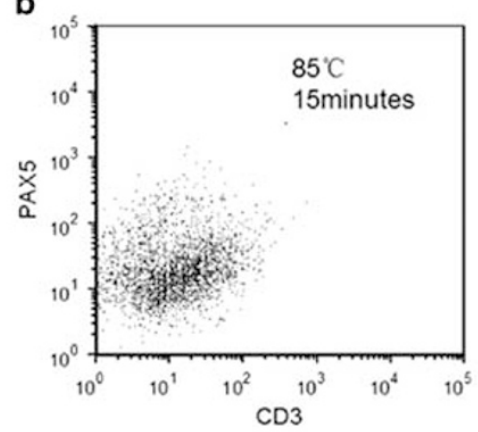

e

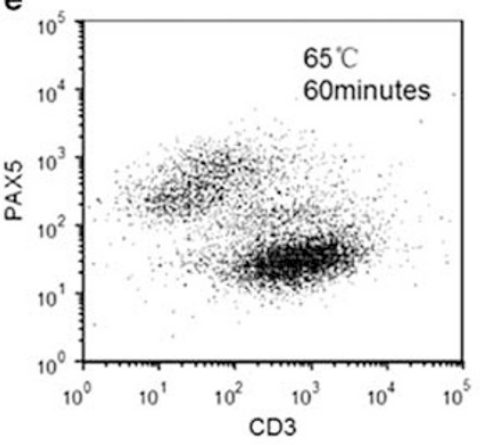

h

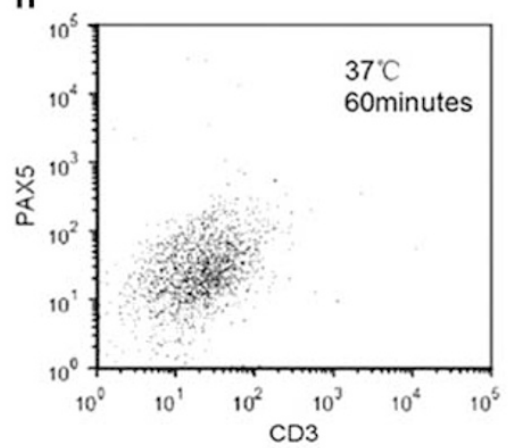

C

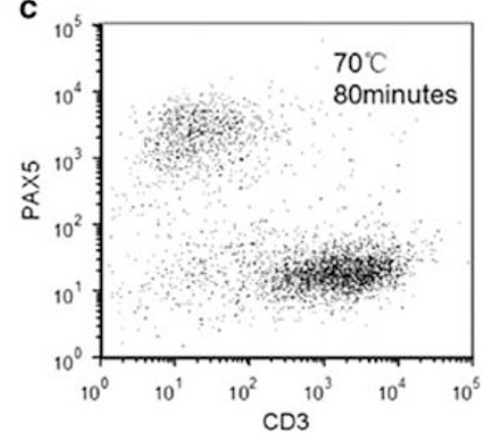

f

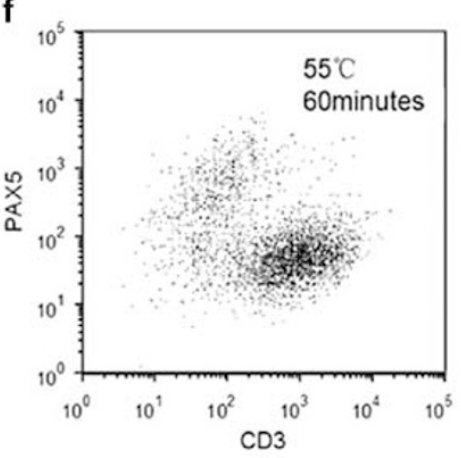

i

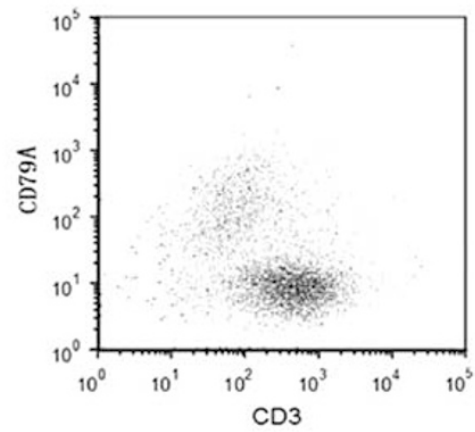

Figure 2 Identification of cell populations from lymphoid tissue with reactive hyperplasia by flow cytometric immunophenotyping using different antigen retrieval conditions. (a, $\mathbf{c}-\mathbf{g}$ ) PAX5-positive cells (B cells) and CD3-positive cells (T cells) were separated as shown on dot plots. Antigen retrieval time and temperature are indicated on the top right corner of each panel. (b, h) Antigens could not be labeled in conditions with short retrieval time or low temperature. For LAT and CD79A labeling, only results using the retrieval condition of $65^{\circ} \mathrm{C} / 60 \mathrm{~min}$ are shown (i, j). 
a

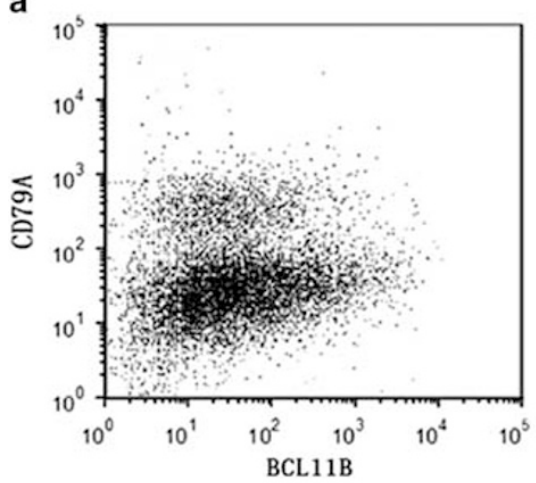

d

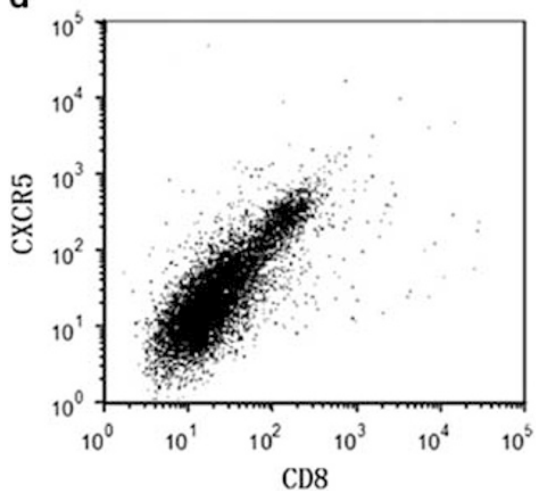

b

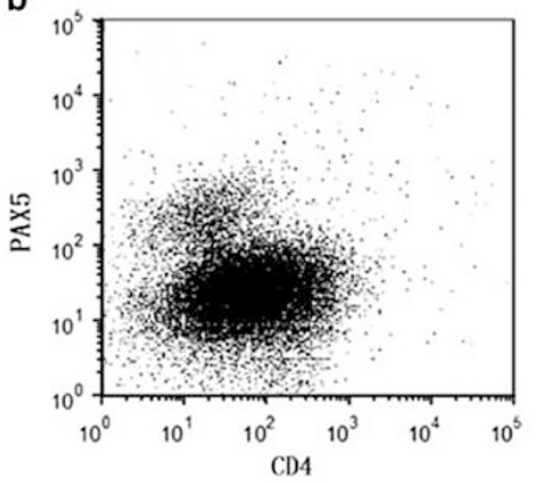

e

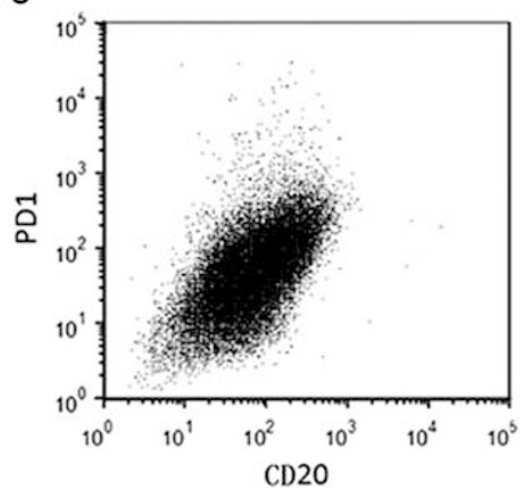

C

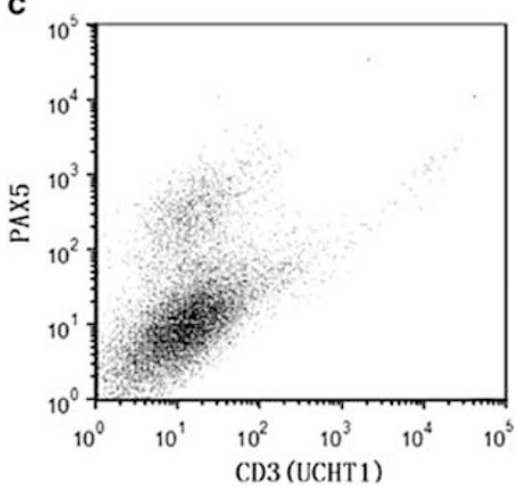

Figure 3 Detection of lymphocyte-specific markers by flow cytometry after antigen retrieval at $65^{\circ} \mathrm{C} / 60 \mathrm{~min}$ in a case of reactive hyperplasia. BCL11B and CD4 were partially and dimly positive (a, b); CD3 (UCHT1), CD8, CD20 and CXCR5 could not be labeled (c-e). PD1 was positive on a small fraction of cells (e).

a

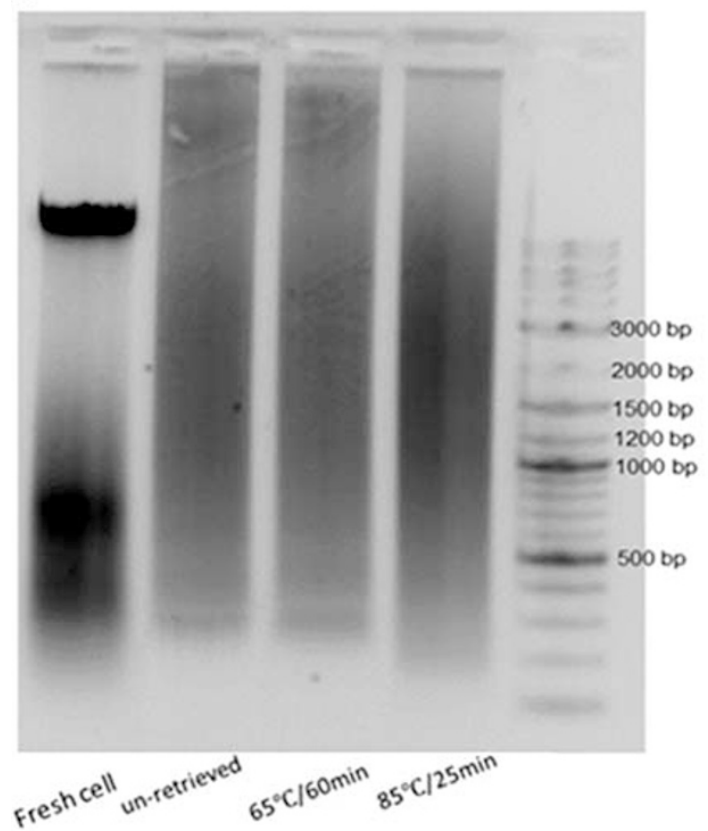

b

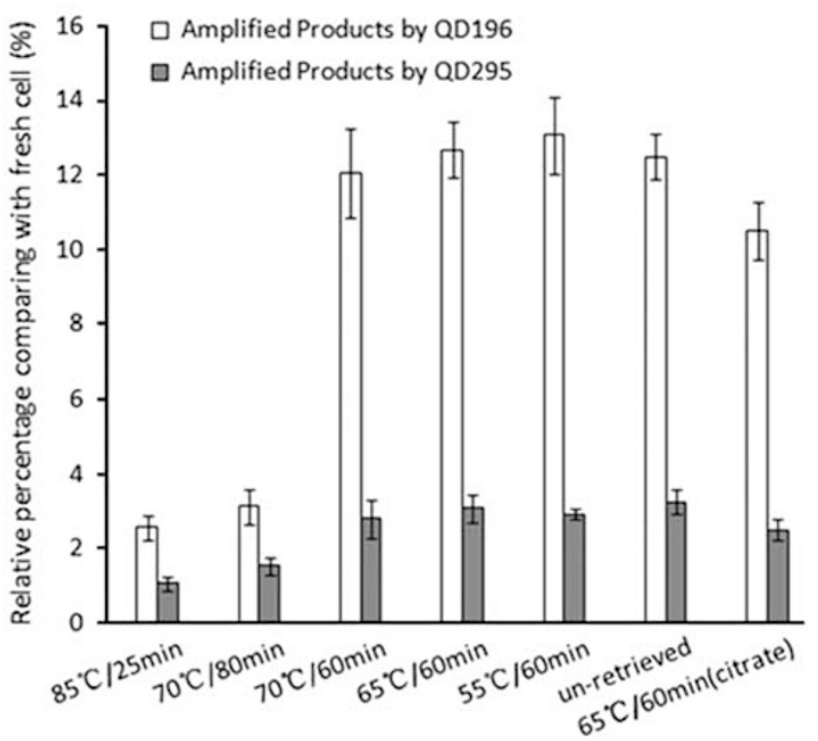

Figure 4 DNA electrophoresis (a) and percentage of amplification product compared to fresh cell line (in three experiments) (b). The qPCR products were obtained after TE buffer antigen retrieval, except the last one. The amplified products of both 196 and 295 bp from FFPE tissue after antigen retrieval under higher temperature or longer incubation, $85^{\circ} \mathrm{C} / 25 \mathrm{~min}$ or $70^{\circ} \mathrm{C} / 80 \mathrm{~min}$, were obviously lower than those with other conditions. In addition, the amplified products of DNA after citrate buffer antigen retrieval were a little lower than after TE buffer. 
DNA fragmentation and degradation. Quantities of amplified products following conditions of high temperature $\left(85^{\circ} \mathrm{C} / 25 \mathrm{~min}\right)$ or long incubation time $\left(70^{\circ} \mathrm{C} / 80 \mathrm{~min}\right)$ were obviously lower than with other antigen retrieval conditions. The same trend was observed for amplified products of $295 \mathrm{bp}$. Incubation at $55-70{ }^{\circ} \mathrm{C}$ for $60 \mathrm{~min}$ seemed to be the optimal temperature-time combination for antigen retrieval while also reducing genomic DNA damage compared with un-retrieved samples. Therefore, $65^{\circ} \mathrm{C} / 60 \mathrm{~min}$ was selected for further studies. In addition, DNA quality is better with $\mathrm{pH}$ 8.0 TE buffer antigen retrieval than with citrate buffer (Figure 4).

Similar observations were made on DNA quality assessed by Bioanalyzer 2100 (Agilent Technologies). The quality of DNA of samples obtained from FFPE tissues without antigen retrieval and with antigen retrieval at $65^{\circ} \mathrm{C} / 60$ min was better than that obtained from FFPE tissue with antigen retrieval at $85^{\circ} \mathrm{C} / 25$ min (Supplementary Figure 3 ). These results suggest that the conditions used in our protocol were optimal for antigen retrieval and flow sorting, without obvious additional negative effect on DNA quality.

DNA recovery with this optimized condition was estimated. In three experiments, the DNA yield per million cells after antibody labeling and flow analysis was 1.8, 2.3 and $2.7 \mu \mathrm{g}$. For further analysis, about 50000 sorted cells were used and yielded at least $100 \mathrm{ng}$ DNA, which is sufficient for NGS.

\section{Sequencing Results}

Lymphoma samples were treated with the optimized conditions. A single-cell suspension from the FL case was labeled with antibodies to CD79A and CD3 and sorted into B-cell and T-cell populations (59\% and $40 \%$, respectively). In immunohistochemical staining, it was estimated that there were $\sim 70 \% \mathrm{~B}$ cells and $30 \% \mathrm{~T}$ cells. The purity of the two populations was then confirmed by immunophenotyping with PAX5 and LAT (Figure 5). DNA from these two cell populations was extracted and sequenced with a custom gene panel. After filtering variants, candidate mutations and VAFs were determined (Supplementary Table 2). True mutations were those found in much higher frequencies in tumor cells as compared to normal cells. Variants present in both of these cell populations at similar VAF (about 50\%) could be excluded as somatic mutations and deemed germline variants. ${ }^{19}$ For the low frequency variants shared between the tumor and non-tumor fractions, we specifically checked the sequence data manually with Integrative Genomics Viewer (IGV) and found most of them to be alignment artifacts (Supplementary Figure 4). In this way, 28 true somatic mutations were identified (Figure $5 \mathrm{~d}$ ). Some of these mutated genes (TNFRSF14, FMN2, ZNF608, KMT2D, CREBBP, BCL2, MEF2B, IRF8, ATM, B2M) have been reported as recurrent mutations with frequencies of close to or $>10 \% .{ }^{20-24}$ Other mutations (RFTN1, TNIK, TET2, NOTCH1, FAS, GNA13, DDX3X, TAF1) have been reported with relatively lower frequencies in $\mathrm{FL}$ and transformed FL. ${ }^{25-30}$ Three mutations (MDN1, LRRN3, ZFP36L1) have been reported only in DLBCL. ${ }^{31,32}$ Two mutations (CUX1, HUWE1) have not been reported in FL or DLBCL.

Cells from two AITL cases were separated into CD3+/PD1 - , $\mathrm{CD} 3+/ \mathrm{PD} 1+$, and $\mathrm{CD} 3-/ \mathrm{PD} 1-$ populations (Figure $6 \mathrm{a})$. The $\mathrm{CD} 3+/ \mathrm{PD} 1+$ population was considered to be tumor $\mathrm{T}$ cells because of the documented PD1 positivity in the lymphoma samples, as is typical for AITL. ${ }^{33}$ In AITL case 1, CD3+/PD1 and $\mathrm{CD} 3+/ \mathrm{PD} 1+$ populations were $47 \%$ and $16 \%$, respectively (about $70 \%$ CD3 + cells by immunohistochemical staining). Extracted DNA was sequenced by NGS (Supplementary Tables 3 and 4). The tumor population of AITL case 1 was found to contain two TET2 mutations, ie TET2-E1320 and TET2-R1516, whereas the $\mathrm{CD} 3+/ \mathrm{PD} 1-$ and $\mathrm{CD} 3-/ \mathrm{PD} 1-$ populations showed only very low TET2 VAF (Figure 6b). In AITL case 2, $\mathrm{CD} 3+/ \mathrm{PD} 1-$ and $\mathrm{CD} 3+/ \mathrm{PD} 1+$ populations were $38 \%$ and $31 \%$, respectively (about $80 \% \mathrm{CD} 3+$ cells by immunohistochemical staining). RHOA, TET2, KMT2C, DYNC1H1, and $\mathrm{IDH} 2$ mutations were confirmed to be associated with the CD3 $+/ \mathrm{PD} 1+$ population with much higher VAF than the CD3 +/PD1 - counterpart (Figure 6c). RHOA, TET2 and IDH2 have been reported as frequent mutations in AITL, ${ }^{34,35}$ while KMT2C mutation has been reported at low frequency. ${ }^{34}$ DYNC1H1 (with VAF of $11.7 \%$ in our study) has not previously been reported in AITL. We sequenced the unseparated tumor tissue with the original block of AITL case 2 and compared the mutations detected with the separated tumor cells. The same somatic mutations were detected with VAFs that were about half of those detected from CD3+/PD1+ cells (Figure 6c), indicating that the tumor cells were enriched by sorting. The frequencies of other SNVs, either germline or artifactual, were similar between $\mathrm{CD} 3+/ \mathrm{PD} 1+$ and $\mathrm{CD} 3$ +/PD1 - cells. Furthermore, we did not detect any new somatic mutations, suggesting that the manipulations associated with the isolation procedure did not alter the mutation profile of the original tumor.

\section{DISCUSSION}

The goal of the present study is to isolate separate populations of neoplastic and normal cells from FFPE tissues so that comparative studies can be performed. There are several challenges that need to be overcome to obtain a robust approach to achieve this goal. Single-cell suspensions need to be prepared with sufficient preservation of structural integrity for subsequent manipulations. In our study, a collagenase and dispase mixture provided a sufficient yield of reasonably intact single cells, but it is important to verify the effect of enzyme digestion, as excessive digestion can lead to weaker staining of some cytoplasmic markers.

The single-cell suspension needs to be labeled for sorting to identify specific populations. Antibody labeling of FFPE tissue generally requires antigen retrieval, and routine antigen retrieval is a harsh process with marked degradation of cellular DNA. We assessed various antigen retrieval 
a

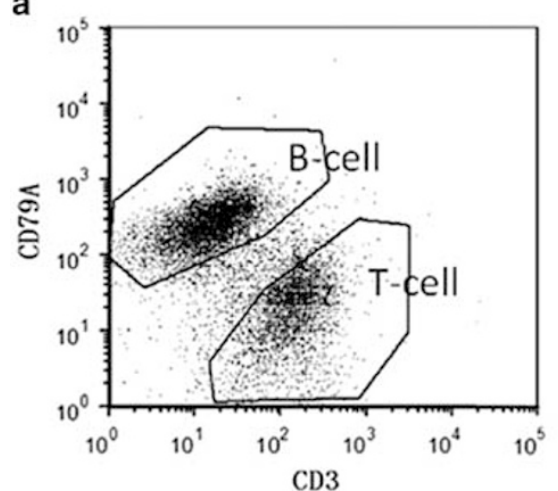

b

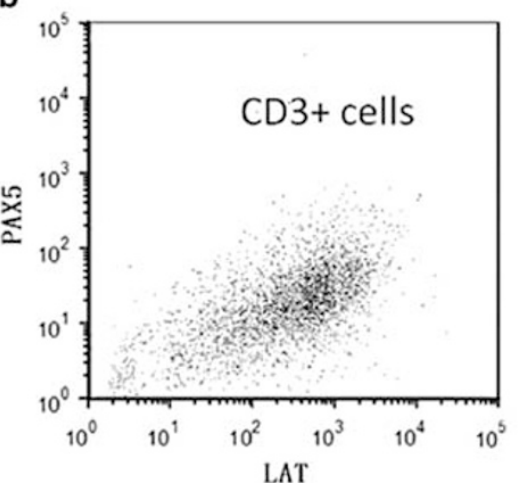

c

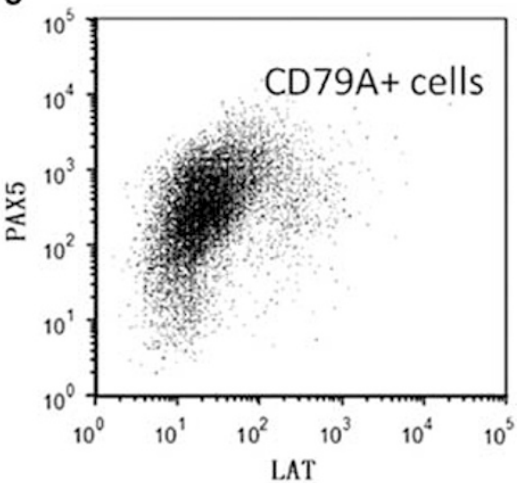

d

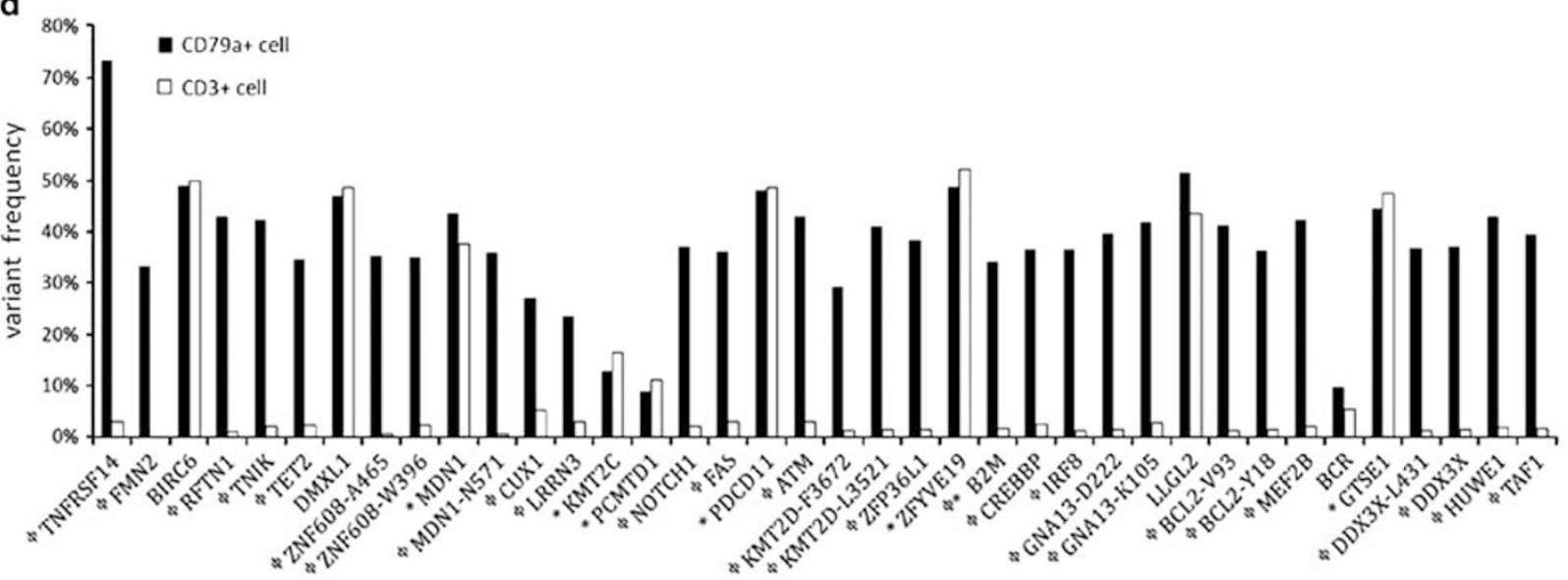

Figure 5 Cell sorting and sequencing for FL case. Single cells from FFPE tissue were separated into B and T cell populations by CD79A and CD3 (a). The sorted cells were confirmed by LAT and PAX5 labeling (b, c). DNA extracted from sorted cells was sequenced, and variants were identified and compared between B-cell and T-cell fractions (d). $\phi$ True somatic mutation. ${ }^{*}$ Variant recorded in dbSNP with population frequency lower than $1 \%$. Some are proven pathogenic mutations in which case both are indicated against the gene. The Low VAF SNP present in all cellular fractions are probably artifacts containing that SNP.

conditions for sufficient staining and optimal preservation of DNA. It has been reported that $1 \mathrm{mM}$ EDTA or Tris- $\mathrm{HCl}$ buffer $(\mathrm{pH} 8.0)$ was more effective than citrate buffer $(\mathrm{pH}$ 6.0) for antigen retrieval, ${ }^{36}$ and by utilizing an alkaline solution for heat-induced retrieval, DNA without further degradation could be extracted. ${ }^{37}$ Therefore, Tris-EDTA buffer with $\mathrm{pH} 8.0$ was used for subsequent antigen retrieval experiments. We found that an antigen retrieval condition of $65^{\circ} \mathrm{C} / 60 \mathrm{~min}$ is suitable for many of the antigen markers we tested. We then compared the DNA quality of samples before and after this antigen retrieval condition and demonstrated that the DNA was of similar quality. Prior studies have indicated that the DNA quality of FFPE tissues is inherently much lower than that DNA extracted from fresh cells under identical conditions. ${ }^{38}$ Our quality assessment using qPCR and the Bioanalyzer indicated that DNA is still of sufficient quality for downstream applications including NGS.

It is essential to determine which antigen markers were preserved for antibody recognition for flow cytometric analysis and sorting. Although the number of analyzed markers was small, we found that cytoplasmic or nuclear antigens were more likely to be maintained and effectively labeled by their corresponding antibodies. However, cell surface markers showed variable and generally weaker results with regard to retrieval and labeling. Because most proteins on the cell surface are transmembrane molecules, the likelihood of the antigen to be maintained and labeled may be related to the epitope's location within the peptide chain. Antigenic determinants located on the ectodomain may be more readily lost in the single cell preparation step, whereas those located near the membrane or facing the cytoplasm may be more likely to be maintained. However, the suitability of an antigen/antibody pair for this purpose needs to be empirically determined, and it is important to indicate precisely which antibody clone (for monoclonal antibodies) is effective. In this study, we have identified a number of T- and B-cell markers whose antibodies worked in our protocol.

Exon capture sequencing using DNA isolated from FFPE samples is technically challenging due to the limited quantity of DNA extracted from these tissues. Sequence alterations and errors could be caused by formalin fixation and DNA crosslinking. ${ }^{39,40}$ False positivities due to sequencing artifact 

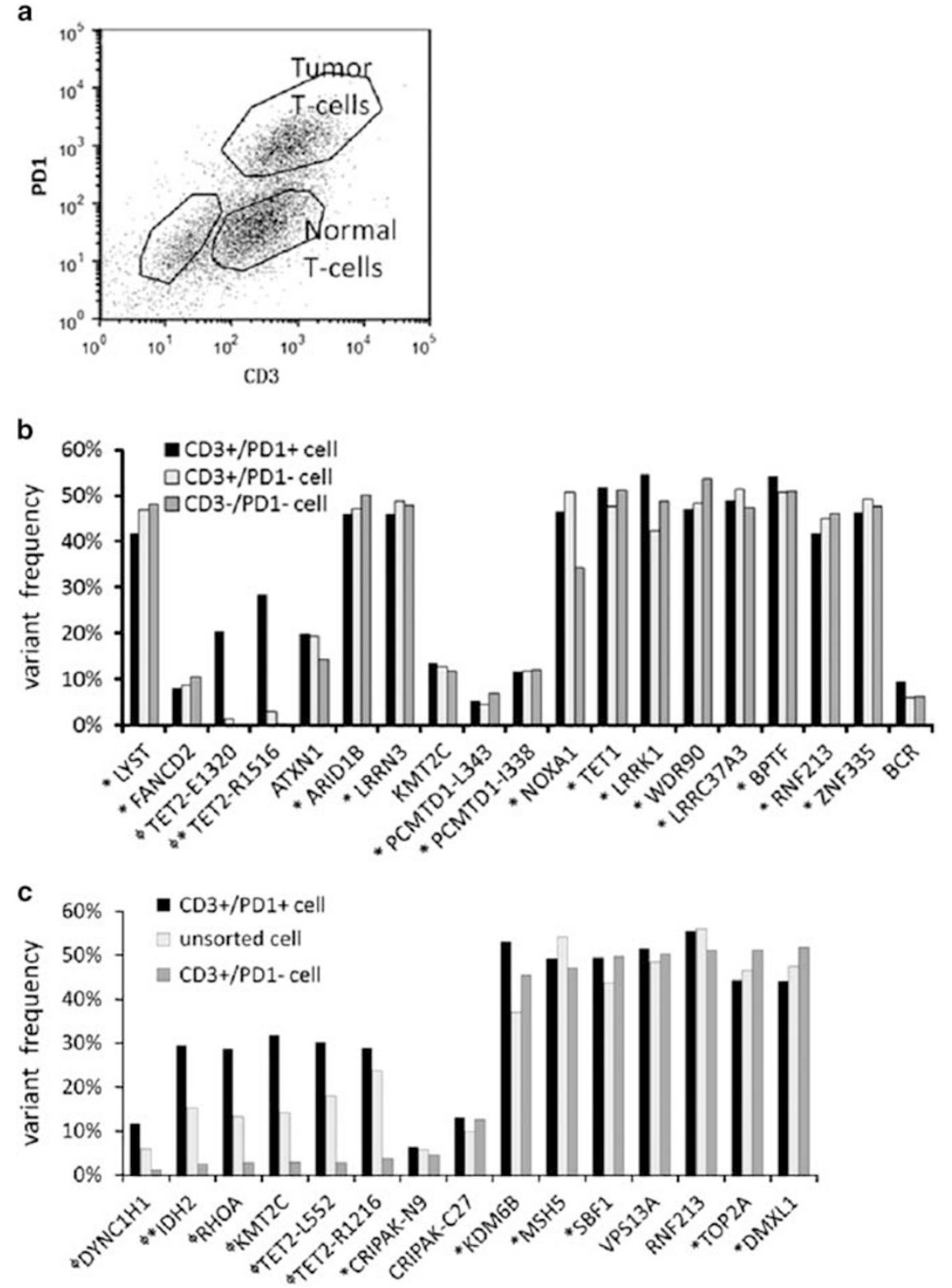

Figure 6 Cell sorting and sequencing for two AITL cases. (a) Cells separated from AITL cases were sorted into CD3+/PD1+, CD3+/PD1 - , and CD3 - /PD1 - populations. (b) DNA extracted from sorted cells was sequenced, and variants were identified. (c) DNA extracted from sorted cells and unseparated tissue of case 2 was sequenced, and variants were identified and compared. $\phi$ True somatic mutation. ${ }^{*}$ Variant recorded in dbSNP with population frequency lower than $1 \%$. Some are proven pathogenic mutations in which case both are indicated against the gene. The Low VAF SNP present in all cellular fractions are probably artifacts containing that SNP.

in DNA from FFPE tissue have been reported. ${ }^{41-43}$ Some studies $^{38,44}$ have compared the results of paired fresh frozen and archival FFPE tissue. The results indicated that although high-confidence mutation calls in the FFPE samples are comparable to that in the frozen samples, caution should be exercised in terms of the artifact due to the background DNA damage of FFPE sample. We therefore tested three tumor samples using a custom capture platform that has worked well in our laboratory. The NGS results from the purified nonneoplastic population derived from the lymphoma sample served as the germline control which we could use to exclude germline variants and sequencing artifacts. Thus, the true lymphoma somatic mutations can be confidently determined, even in samples without a corresponding normal control. It can be seen that even with a limited panel of lymphoma associated genes, there are germline SNVs that are not readily screened out without the help of the 'normal' population. Although most of the variants recorded in dbSNP are indeed germline variants, several are actually known pathogenic mutations. Conversely, some germline variants are not present in dbSNP, and are known as private SNPs. There were also some shared variants with low VAF in both tumor 
and non-tumor fractions. Most of these variants represented artifacts resulted from false calls because some variant reads were actually from paralogous or repeat genomic regions that were mapped to the wrong genomic position. These artifacts might be erroneously considered true somatic mutations if only the unsorted tissue was sequenced.

AITL is notoriously difficult to study by NGS, as the tumor content is frequently low, and tumor cells may not be easy to separate from reactive T-cells in the background.$^{45}$ We have demonstrated that we can substantially enrich the tumor cell population, and the tumor-depleted population could be used effectively as the germline control. Almost all of the SNVs detected that showed a large difference in VAF between the tumor and non-tumor population are known mutations in AITL. ${ }^{34,35}$

Our study demonstrated that it is possible to obtain singlecell suspensions from FFPE tissue and to obtain specific cellular populations through multi-parameter flow cytometric sorting. We have tested different antigen retrieval conditions and a number of antibodies to antigens in different cellular locations and have provided an optimized protocol for the procedure. We have also demonstrated the utility of this procedure in NGS for investigating gene mutations present in lymphoma samples containing heterogeneous components. Additional antibodies should be evaluated for labeling an extended range of antigen markers especially the labeling of membrane markers. This approach has the potential to unlock the huge archives of FFPE tissues for NGS investigations. This protocol can also be further modified for broader applications including single-cell assays and the objective measurement of non-malignant cells in the tumor microenvironment by flow cytometry. ${ }^{46}$

Supplementary Information accompanies the paper on the Laboratory Investigation website (http://www.laboratoryinvestigation.org)

\section{ACKNOWLEDGMENTS}

We thank Ni Feng and Lucy Brown of the Analytical Cytometry Core for flow cytometric sorting, and Lewallen Michael of the Research Pathology Core for tissue section preparation. We are grateful for help from Jinhui Wang and Dr Xiwei Wu of the Integrative Genomics Core for next-generation sequencing. Research reported in this publication was supported by the City of Hope Lymphoma Specialized Programs of Research Excellence (SPORE) grant P50CA107399 and the National Cancer Institute of the National Institutes of Health under award number P30CA033572. The content is solely the responsibility of the authors and does not necessarily represent the official views of the National Institutes of Health.

\section{DISCLOSURE/CONFLICT OF INTEREST}

The authors declare no conflict of interest.

1. Killian JK, Bilke $S$, Davis $S$, et al. Large-scale profiling of archival lymph nodes reveals pervasive remodeling of the follicular lymphoma methylome. Cancer Res 2009;69:758-764.

2. Alioto TS, Buchhalter I, Derdak S, et al. A comprehensive assessment of somatic mutation detection in cancer using whole-genome sequencing. Nat Commun 2015;6:10001.
3. Wei L, Papanicolau-Sengos A, Liu S, et al. Pitfalls of improperly procured adjacent non-neoplastic tissue for somatic mutation analysis using next-generation sequencing. BMC Med Genomics 2016;9:64.

4. Kassahn $\mathrm{KS}$, Holmes $\mathrm{O}$, Nones $\mathrm{K}$, et al. Somatic point mutation calling in low cellularity tumors. PLoS One 2013;8:e74380.

5. Fromm JR, Wood BL. Strategies for immunophenotyping and purifying classical Hodgkin lymphoma cells from lymph nodes by flow cytometry and flow cytometric cell sorting. Methods 2012;57: 368-375.

6. Reichel J, Chadburn A, Rubinstein PG, et al. Flow sorting and exome sequencing reveal the oncogenome of primary Hodgkin and ReedSternberg cells. Blood 2015;125:1061-1072.

7. Muschen M, Kuppers R, Spieker T, et al. Molecular single-cell analysis of Hodgkin- and Reed-Sternberg cells harboring unmutated immunoglobulin variable region genes. Lab Invest 2001;81:289-295.

8. Delabie J, Tierens A, Wu G, et al. Lymphocyte predominance Hodgkin's disease: lineage and clonality determination using a single-cell assay. Blood 1994;84:3291-3298.

9. Gross A, Schoendube J, Zimmermann $S$, et al. Technologies for Single-Cell Isolation. Int J Mol Sci 2015;16:16897-16919.

10. Kuppers R, Schneider M, Hansmann ML. Laser-based microdissection of single cells from tissue sections and PCR analysis of rearranged immunoglobulin genes from isolated normal and malignant human B cells. Methods Mol Biol 2013;971:49-63.

11. Peveling-Oberhag J, Wolters F, Doring $C$, et al. Whole exome sequencing of microdissected splenic marginal zone lymphoma: a study to discover novel tumor-specific mutations. BMC Cancer 2015;15:773.

12. Johnson NA, Hamoudi RA, Ichimura $\mathrm{K}$, et al. Application of array $\mathrm{CGH}$ on archival formalin-fixed paraffin-embedded tissues including small numbers of microdissected cells. Lab Invest 2006;86: 968-978.

13. Bolognesi C, Forcato C, Buson G, et al. Digital sorting of pure cell populations enables unambiguous genetic analysis of heterogeneous formalin-fixed paraffin-embedded tumors by next generation sequencing. Sci Rep 2016;6:20944.

14. Jordanova ES, Corver WE, Vonk MJ, et al. Flow cytometric sorting of paraffin-embedded tumor tissues considerably improves molecular genetic analysis. Am J Clin Pathol 2003;120:327-334.

15. Li H, Durbin R. Fast and accurate short read alignment with BurrowsWheeler transform. Bioinformatics (Oxford, England) 2009;25: 1754-1760.

16. McKenna A, Hanna M, Banks E, et al. The Genome Analysis Toolkit: a MapReduce framework for analyzing next-generation DNA sequencing data. Genome Res 2010;20:1297-1303.

17. Koboldt $\mathrm{DC}$, Zhang $\mathrm{Q}$, Larson $\mathrm{DE}$, et al. VarScan 2: somatic mutation and copy number alteration discovery in cancer by exome sequencing. Genome Res 2012;22:568-576.

18. Wang K, Li M, Hakonarson H. ANNOVAR: functional annotation of genetic variants from high-throughput sequencing data. Nucleic Acids Res 2010;38:e164.

19. Wong SQ, Li J, Tan AYC, et al. Sequence artefacts in a prospective series of formalin-fixed tumours tested for mutations in hotspot regions by massively parallel sequencing. BMC Medical Genomics 2014;7:23.

20. Correia C, Schneider PA, Dai H, et al. BCL2 mutations are associated with increased risk of transformation and shortened survival in follicular lymphoma. Blood 2015;125:658-667.

21. Sarkin E, Jaffe DAA, Campo E, et al. Hematopathology, 2nd edn. Elsevier Health Sciences, 2016, p 82.

22. Kridel R, Sehn LH, Gascoyne RD. Pathogenesis of follicular lymphoma. J Clin Invest 2012;122:3424-3431.

23. Krysiak K, Gomez F, White BS, et al. Recurrent somatic mutations affecting B-cell receptor signaling pathway genes in follicular lymphoma. Blood 2017;129:473-483.

24. Gumy-Pause F, Wacker P, Sappino AP. ATM gene and lymphoid malignancies. Leukemia 2004;18:238-242.

25. Lossos IS, Gascoyne RD. Transformation of follicular lymphoma. Best Pract Res Clin Haematol 2011;24:147-163.

26. Karube K, Martinez D, Royo $C$, et al. Recurrent mutations of $\mathrm{NOTCH}$ genes in follicular lymphoma identify a distinctive subset of tumours. J Pathol 2014;234:423-430.

27. Pastore A, Jurinovic V, Kridel $R$, et al. Integration of gene mutations in risk prognostication for patients receiving first-line immunochemotherapy for follicular lymphoma: a retrospective analysis of a 
prospective clinical trial and validation in a population-based registry. Lancet Oncol 2015;16:1111-1122.

28. Pasqualucci L, Khiabanian $\mathrm{H}$, Fangazio $M$, et al. Genetics of follicular lymphoma transformation. Cell Rep 2014;6:130-140.

29. Bouska A, McKeithan TW, Deffenbacher KE, et al. Genome-wide copynumber analyses reveal genomic abnormalities involved in transformation of follicular lymphoma. Blood 2014;123:1681-1690.

30. Bouska A, Zhang W, Gong Q, et al. Combined copy number and mutation analysis identifies oncogenic pathways associated with transformation of follicular lymphoma. Leukemia 2017;31:83-91.

31. Pasqualucci L, Trifonov V, Fabbri G, et al. Analysis of the coding genome of diffuse large B-cell lymphoma. Nat Genet 2011;43:830-837.

32. De Miranda NF, Georgiou K, Chen L, et al. Exome sequencing reveals novel mutation targets in diffuse large B-cell lymphomas derived from Chinese patients. Blood 2014;124:2544-2553.

33. Roncador G, Garcia Verdes-Montenegro JF, Tedoldi S, et al. Expression of two markers of germinal center T cells (SAP and PD-1) in angioimmunoblastic T-cell lymphoma. Haematologica 2007;92:1059-1066.

34. Yoo HY, Sung MK, Lee $\mathrm{SH}$, et al. A recurrent inactivating mutation in RHOA GTPase in angioimmunoblastic T cell lymphoma. Nat Genet 2014;46:371-375.

35. Odejide $\mathrm{O}$, Weigert $\mathrm{O}$, Lane $\mathrm{AA}$, et al. A targeted mutational landscape of angioimmunoblastic T-cell lymphoma. Blood 2014;123:1293-1296.

36. Pileri SA, Roncador $\mathrm{G}$, Ceccarelli $\mathrm{C}$, et al. Antigen retrieval techniques in immunohistochemistry: comparison of different methods. J Pathol 1997;183:116-123.

37. Shi SR, Datar R, Liu C, et al. DNA extraction from archival formalin-fixed, paraffin-embedded tissues: heat-induced retrieval in alkaline solution. Histochem Cell Biol 2004;122:211-218.
38. Hedegaard J, Thorsen $\mathrm{K}$, Lund MK, et al. Next-generation sequencing of RNA and DNA isolated from paired fresh-frozen and formalin-fixed paraffin-embedded samples of human cancer and normal tissue. PLoS One 2014;9:e98187.

39. Graw $S$, Meier $R$, Minn $K$, et al. Robust gene expression and mutation analyses of RNA-sequencing of formalin-fixed diagnostic tumor samples. Sci Rep 2015;5:12335.

40. Munchel S, Hoang Y, Zhao Y, et al. Targeted or whole genome sequencing of formalin fixed tissue samples: potential applications in cancer genomics. Oncotarget 2015;6:25943-25961.

41. Do H, Dobrovic A. Sequence artifacts in DNA from formalin-fixed tissues: causes and strategies for minimization. Clin Chem 2015;61:64-71.

42. Do H, Wong SQ, Li J, et al. Reducing sequence artifacts in ampliconbased massively parallel sequencing of formalin-fixed paraffinembedded DNA by enzymatic depletion of uracil-containing templates. Clin Chem 2013;59:1376-1383.

43. Wong SQ, Li J, Tan AY-C, et al. Sequence artefacts in a prospective series of formalin-fixed tumours tested for mutations in hotspot regions by massively parallel sequencing. BMC Med Genomics 2014;7: $1-10$.

44. Oh E, Choi YL, Kwon MJ, et al. Comparison of accuracy of whole-exome sequencing with formalin-fixed paraffin-embedded and fresh frozen tissue samples. PLoS ONE 2015;10:e144162.

45. Gaulard P, de Leval L. The microenvironment in T-cell lymphomas: emerging themes. Semin Cancer Biol 2014;24:49-60.

46. Sander B, de Jong D, Rosenwald A, et al. The reliability of immunohistochemical analysis of the tumor microenvironment in follicular lymphoma: a validation study from the Lunenburg Lymphoma Biomarker Consortium. Haematologica 2014;99:715-725. 